\title{
Application of inductive coupling for wireless power transfer
}

\author{
Ibrahim Alhamrouni ${ }^{1}$, M. Iskandar², Mohamed Salem ${ }^{3}$, Lilik J. Awalin ${ }^{4}$, Awang Jusoh ${ }^{5}$, Tole Sutikno ${ }^{6}$ \\ ${ }^{1,2,4}$ Electrical Engineering Section, University Kuala Lumpur (UniKL BMI), Malaysia \\ ${ }^{3}$ School of Electrical and Electronic Engineering, Universiti Sains Malaysia, Malaysia \\ ${ }^{5}$ School of Electrical Engineering, Universiti Teknologi Malaysia, Malaysia \\ ${ }^{6}$ Department of Electrical Engineering, Universitas Ahmad Dahlan, Indonesia
}

\begin{tabular}{l} 
Article Info \\
\hline Article history: \\
Received Jan 4, 2020 \\
Revised Mar 2, 2020 \\
Accepted Mar 29, 2020
\end{tabular}

\section{Keywords:}

Capacitive coupling Inductive coupling Transfer efficiency Wireless power transfer

\begin{abstract}
Considering the massive development that took place in the past two decades, wireless power transfer has yet to show the applicability to be used due to several factors. This work focuses on determining the main parameters like, mutual inductance, and coupling coefficient for a pair of helical coils for wireless power transfer applications. These parameters are important in designing and analyzing a wireless power transfer system based on the phenomenon of inductive/ resonant inductive coupling. Here presents a simple approach based on fundamental laws of physics for determining the coupled coil parameters for single layered helical coils. The results conducted by computer simulation which is MATLAB. Furthermore, this analysis is used to study the effect of change in coil diameter, mutual inductance coefficient and change in distance between coils on parameters like self and mutual inductance of coupled coils which is of great importance in Wireless Power Transfer applications. The research yielded promising results to show that wireless power transfer has huge possibility to solve many existing industrial problems.
\end{abstract}

This is an open access article under the CC BY-SA license.

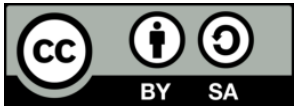

\section{Corresponding Author:}

Ibrahim Alhamrouni,

Electrical Engineering Section,

University Kuala Lumpur British Malaysian Institute (UniKL BMI),

Gombak 53100, Malaysia.

Email: ibrahim.mohamed@unikl.edu.my

\section{INTRODUCTION}

Recently, wireless power transfer has got attention from researchers and inventors because this technology has opportunity to improve lifestyle in this era for human being. To transfer the electrical power from one place to another place, copper cable is usually used as a medium. For the example the fluorescent lamp needs copper cable to carry the current in the other term to carry the power to fluorescent lamp function as well. So, the wireless power gives something new for the electrical appliance like fluorescent lamp which is the new medium to transfer the power without using cable but by using electromagnetic wave or other medium.

In the 20th century Nikola Tesla, the American engineer and inventor attempted the first experiment of wireless power transfer. He successfully transferred the power from the tower, which is Wardenclyffe Tower in shoreham to bright up the bulb in a few meters. This has proved that the idea of transmitting the power wirelessly had been a long time ago [1-3]. As a next generation, the need to understand and improve the capability of wireless power transmission in the next level in term of increasing the distance and the efficiency of the overall system. 
The cost to make the copper cable and to install it will hit a lot of numbers and will be quite expensive. For the example, the undersea cable which is costly and dangerous in many cases. This problem needs to be faced because of the demand of the power not only at the urban place but also in the rural ones. There are a lot of places in the world that has no electric power because of the limitation of cable and some other technical issues [4-6].

With the rapid increase of electricity demand and the obstacle of supplying the power to certain places and condition, new technique to transfer the power had to be introduced to replace the conventional ones. Wireless power transfer is considered as the concept that has the opportunity. Recently, wireless power transfer is the new term defined by researchers and massive effort has been put on to advance such technique. Although, it has been available based on previous studies by the researchers and some of electric appliance are already using that concept, this work focuses on the design and method of wireless power transfer application $[7,8]$.

\section{WIRELESS POWER TRANSFER METHODS}

In 1891, one of the famous American engineers, scientist and inventor that is Nikola Tesla tried to prove that transferring the power to the load without using any cable by using the electro dynamic induction concept and air as a medium to light up the light bulb. An experiment was conducted at lab in Colorado USA, he built a small tower coil as a source of the power and lighted a bulb as a load, he succeeded to light up the bulb a few meters. Some researchers have come up with their own research and data to improve the ability of the system [9]. Some of the researchers used other concept like capacitive and transfer by microwave.

There are several methods that have been used by the researchers to improve the system itself. Inductive coupling, resonant inductive coupling, capacitive coupling and microwave. All of these methods have been divided into two groups which is radiative and non-radioactive, all of them also have different concept and different result from the distance and efficiency but all of those method have the same objective which is to transfer the power from the source to load without using any cable [10, 11].

\subsection{Inductive coupling}

Inductive coupling is one of the methods that have been used in wireless power transfer system. Among all of the methods, this is the simplest method, this concept is very simple to understand because it uses the mutual induction concept just like electrical transformers. In this system, it uses two separate coils which is L1 as first coil and L2 which is the second coil. The first coil L1 will attach to the source so it becomes transmitter and the second coil L2 will attach with the load therefore it becomes the receiver [12-14].

The power will transmit from the L1 to L2 by electromagnetic wave that was created by the first coil. This is because when the current goes through conductor (coil) the magnetic flux will be produced. So, the magnetic flux that has been produced from the first coil, will move to the second coil L2 and cut the coil. From basic electromagnetic induction, when the magnetic flux cut the conductor, electric current will induce. After that, the current will flow to the load as an AC current. The rectifier will convert the AC current to the DC current if the load requires a DC supply [15]. Figure 1 as shown the block diagram of wireless power transfer induction coupling method.

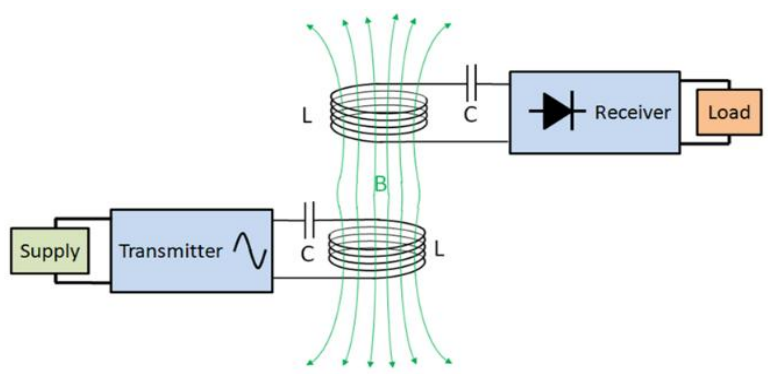

Figure 1. The block diagram of wireless power transfer induction coupling method

\subsection{Resonance inductive coupling}

Resonant inductive coupling or the electro inductive effect is better than the inductive coupling. By using this method, more power can be transferred between the two coils which are L1 and L2, longer in 
distance and efficiently by using the concept of resonance. Every object in universe has their own natural frequency, if the natural frequency of objects is matched, resonance phenomena will occur [16]. During this phenomenon, the amplitude will vibrate at maximum amplitude which means that maximum amount of energy will transfer to the object.

By this method, the electromagnetic wave will not be absorbed or wasted because the wave is not propagating through the air for being that. MIIT researchers have developed a prototype of resonant inductive coupling method that can transfer the power up to 5-meter range [17]. According to the researchers, it will not disrupt other electronic devices or cause injuries to the living things. In other words, it is safe to environment and can ease the job of many industries and reduce the cost [18].

In resonant inductive coupling method, two separate coil which are transmitter coil and receiver coil are tuned at the same resonant frequency. The electric power that produce at the receiver coil depends on the concept of mutual inductance, varying magnetic field that have been formed by transmitter coil. The varying magnetic field that was formed will cut the receiver coil and current will be induced at the receiver coil.

To increase the efficiency of this system, the Q factor must be increased at the LC circuit. The $\mathrm{Q}$ factor would be increased by lowering the resistance or capacitance value. The value of resistance can be lowered by using the high quality of winding wire for the coil or by using thicker wire [19-21].

$$
Q=\frac{1}{R} \sqrt{\frac{L}{C}}
$$

This method has several advantages such as, high efficiency because of the resonant effect that allows the high vibrate of amplitude which means the maximum energy will be transferred through it. By using this method also, the power can be transferred through longer distance compared to non-resonant inductive coupling. The magnetic field also has no effect by surrounding the objects around it. They can be transferred through barriers like plastic, wood and glass. This method is also safe for human, animal and surrounding environment because there is no electromagnetic radiation during the power transfer from the source to the load $[11,22,23]$.

\subsection{Wireless power transmission using microwaves}

Transferring the power by using microwave is categorized as radiative. This method is also called far-field technique, because by using this method, power can be transferred for longer distance by beams of electromagnetic radiation such as microwave. This method was pioneered by William C. Brown and demonstrated that power transferred through free space by microwave [24].

From the transmitter, the power will be converted to the form of microwave by microwave generator, transmitting antenna will propagate the signal to the free space. For the high aperture efficiency $(>95 \%)$, ideal slotted waveguide antenna is normally used, it also gives high power handling capability [20]. The receiver will receive the microwave signal, a special device which is rectenna works as a harvester. Rectenna harvests energy of electric from the microwave signal that has been propagated by the transmitter. Schottky diodes have been used in rectifier because it has the ability of faster reverse recovery time and good for RF characteristic, DC supply will be produced and can stored in the energy storage. Microwaves frequencies from $1 \mathrm{GHz}$ up to $1000 \mathrm{GHz}$ have been used in this method.

In 1980, the first practical application of microwave power transmission was introduced by Canada's communication research Centre. Unmanned airplane that power microwave beam from the ground and was used as a communication relay which can fly 2 kilometers in a circle diameter at altitude 21 kilometer. The plane name stationary high-altitude relay platform or known as (SHARP) can fly for month without landing [25].Shortcomings of this system or method, the first implementation cost for practical wireless microwave power transmission reasonably is too expensive. Telecommunication today such as radio, television and others use microwave as a medium signal. This concept will intervene with the present communication signal system because it also uses microwave to transfer the power from transmitter to the receiver. From the biological impact, common believe that, the effect of radiation signal cause several problems and have serious consequences. But the dose of radiation dose that receive from microwave signal lower than while opening the microwave oven door [25].

\subsection{Electrostatic induction}

The capacitive charging method is a technique of transferring the energy through two barriers plate that close contact to one another. Figure 2 below shows the structure of a capacitive power transfer that consists of inverter, primary side, capacitive coupler, secondary side, rectifier and load [26, 27]. 


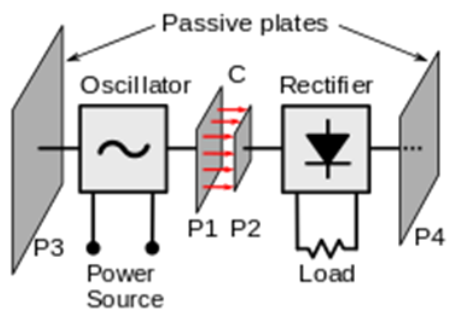

Figure 2. The concept of electrostatic induction

The dc voltage from the source converted into alternating current (AC) voltage using high frequency inverter. Afterwards it supplies it to two primary metal plates. There are four metal plate required to form capacitive coupling. Two metal plate at primary side as power transmitter and two metal plate at secondary side as power receiver. The interlink between the two secondary metal plates that located adjacent with the two primary metal plates generate the electric field that enable displacement of the current to flow [10].

The current receiving through the secondary side and converted to direct current (DC) voltage using rectifier. The capacitive coupling contributes to the stability of operation of capacitive power transfer. The value of capacitive coupler depends on distance, area of plate and material of dielectric. The application of capacitive coupling power transfer limited for short distance and low voltage application such mobile phone charging, LED lighting, USB and biomedical devices. The power capacitive coupling increased to $\mathrm{kW}$ level.

\section{PROPOSED DESIGN}

Wireless power transfer is the system that transfer the power from source to the load without physical contact between them. This work had to be introduced due of the lack and the problem of transferring the power based on condition of the place. This part focuses on the design of the wireless power transfer to solve the raising problems that currently occur when transfer power wirelessly.

Based on all the method that has been discussed in the previous part, wireless power transfer based on inductive coupling has been selected based on criteria, advantage and limitation with respect to other methods.

To transfer the power wirelessly by inductive coupling, the principle of magnetic wave needs to be applied in this method to set corresponding between transmitting and receiving part with related parameters. The magnetic wave of transmitter and receiver must be in the same resonant frequency to achieve maximum transfer of power exchange between them. Therefore, coupling transmitter coil and receiver coil of the circuit are placed in proximity to one another such that transmitter can transfer the power to the receiver efficiently.

The characteristic of the coil at the transmitter and receiver contribute in term of efficiency of the system to transfer the power from the source to the load. The efficiency can be increased by increasing the number of turns of coil, diameter of coil, the cross-sectional area of the coil, the strength of current and the strength of the radial magnetic field that produced by the coil. For this research, the air core coil is the inductor which does not depend on ferromagnetic material to get its specified value of inductance. The air core coil had been chosen based on its advantages, where it has in high frequency, high stability, better efficiency, greater power handling and less distortion. The air core coil also has low resistance towards $\mathrm{DC}$ and linear AC.

The criteria to design the inductive coupling had to consider the number of turns of coil. For this work, a cylindrical coil had been chosen because of the steady state current flow through the coil. The strength of current also need to be considered because it will affect the strength of magnetic field that is produced by the coil. The number of turns, N, radius of coil, r, length of coil winding, current, I, and the depth of the coil, $\mathrm{d}$ can determine the value of inductance, $\mathrm{L}$ by using the formula as shown below.

$$
L=\frac{R^{2} N^{2}}{10 l+9 R}
$$

Where $\mathrm{L}$ is the value of inductance, $\mathrm{R}$ is the value of resistance in coil, $\mathrm{N}$ is the number of turns of coil and $\{$ is the length of the cable winding.

For the single turn circular coil of self-inductance is given by: 


$$
L(\text { one turn })=\frac{\Phi}{i}
$$

Flux one turn is the flux that passing through the inner diameter of circular coil. Assuming that there is no flux leakage between the turns, that means all the turns couple tightly with each other. Self-inductance of coil is given by:

$$
L=N^{2} L(\text { one turn })
$$

For the pair of single mutual inductance coil is given by:

$$
M=N 1 \text { N2Mone turn }
$$

$\mathrm{M}=$ flux produced by primary coil passing through secondary coil current in the secondary Assuming there is no flux leakage. The T-Model as shown in Figure 3.

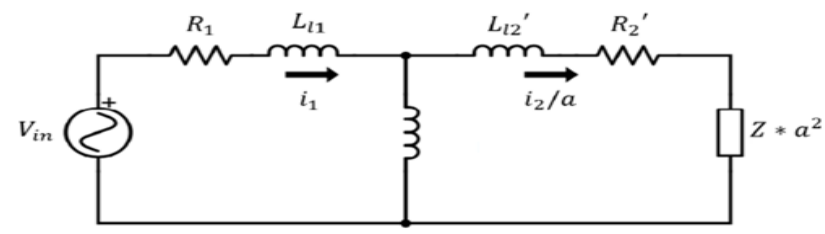

Figure 3. The T-model

Coefficient of mutual induction is defined as the ratio of magnetic flux in receiver coil to the current in transmitter coil. Consider two coils placed very close to each other, the first coil will connect to the voltage source of variable e.m.f or alternating current source. The second coil will connect to the load or to the galvanometer. Coefficient of mutual inductance will depend on the shape and size of the coil. The number of turns each coil has and the angular orientation between coils also play a vital role. Last but not list, the medium between the coil can also affect the value of coefficient between the transmitter and receiver coils.

Coefficient of coupling effects the mutual induction between the two coils by relative orientation of the coil. In other words, the method of joining the two coils effect mutual induction. The amount of flux generated by the first coil is hundred percent linked with the second coil. This supposed to be the best type of coupling. Coefficient of coupling: $K=1$. If we separate the two coils, that means we put the second coil away from the first coil, the amount of flux generated is not hundred percent linked with the second coil, some of it may be lost in the surrounding. When the second coil is perpendicular to the first coil, the amount of flux generated by the first coil is not at all linked with the second coil, all the flux is lost, the coefficient: $\mathrm{k}=0$.

To transfer the electrical power from transmitter coil to the receiver coil, the value of coefficient must be $\mathrm{k}=>1$ to ensure that the power transfer from the transmitter to the receiver is done efficiently. We can get the value $\mathrm{k}$ by using the formula:

$$
k=\frac{1}{\left(1+2^{\frac{2}{3}}\right)\left(\frac{D^{2}}{R x T x}\right)^{\frac{3}{2}}}
$$

\section{RESULTS AND DISCUSSION}

In this part, the simulation by MATLAB Simulink shows and test all the parameter that effect the distance and efficiency of wireless power transfer. From the equation, the value of $\mathrm{k}$ will increase if the product of radius Tx and Rx have been increased. The larger the radius of the transmitter and receiver coil, the power transfer between the coils will occur in more efficient way. The value of $k=1$ if the distance $D$ is equal to zero. It means that there is no gap between the transmitter and receiver coil. But, in term of wireless power transfer, the gap between the transmitter and receiver coils will increase at the same time the efficiency also must be considered. The graph coefficient against product of Tx and Rx as shown in Figure 4 and coefficient and $\mathrm{Tx} * \mathrm{Rx}$ as shown in Table 1. 
Table 1. Coefficient and $\mathrm{Tx} * \mathrm{Rx}$

\begin{tabular}{ccccccccccc}
\hline Coefficient & 0.003 & 0.01 & 0.02 & 0.05 & 0.07 & 0.11 & 0.15 & 0.19 & 0.24 \\
\hline Tx*Rx & 0.04 & 0.09 & 0.16 & 0.25 & 0.36 & 0.49 & 0.64 & 0.81 & 1 \\
\hline
\end{tabular}

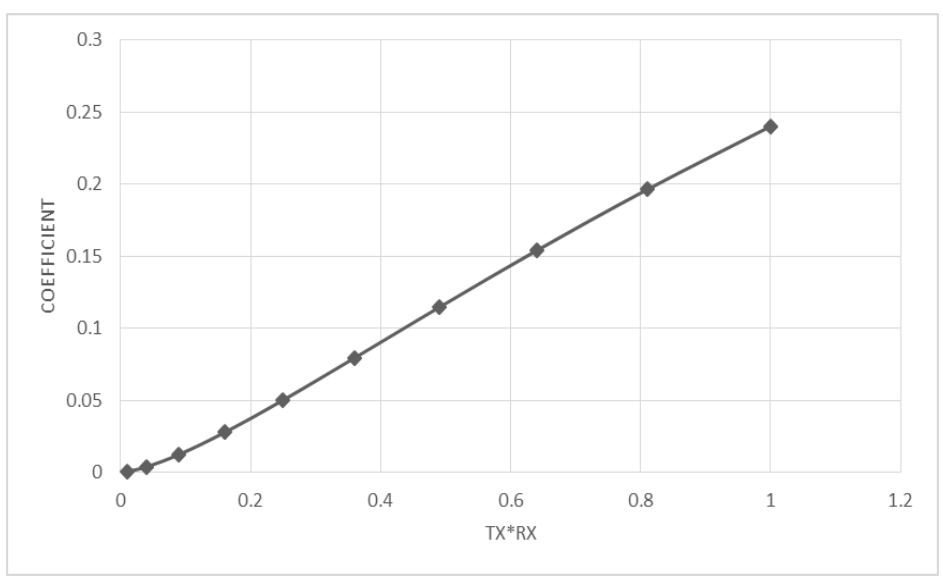

Figure 4. The graph coefficient against product of Tx and Rx

The value of voltage will effect the value of the electromagnetic field which is produced by inductor at the transmitter. The value of electromagnetic field will increase if the value if the voltage increased. When the value of electromagnetic field increases, the wave that produce will go more in term of distance, because it has more energy to transmit the wave. Figure 8 shows the value of voltage source which is 10 volts. The distance between the Rx and Tx is 1 meter and the radius of RxTx is 1 meter.

From the figure above, the value of the voltage was same as the input voltage because of the transformer that is used to pump up back the voltage that lost during the transmission process which is from the transmitter to the receiver. Therefore, the distance between transmitter and receiver, can increase as the efficiency between both of them remains high. To ensure there is no disturbance during the transformation of power between transmitter and receiver coil, the value of frequency of sinusoidal voltage can be increased.

Figure 5 shows the circuit design for wireless power transfer, meanwhile Figures 6 and 7 illustrate the input and out voltage waveforms and coefficient and output voltage as shown in Table 2.

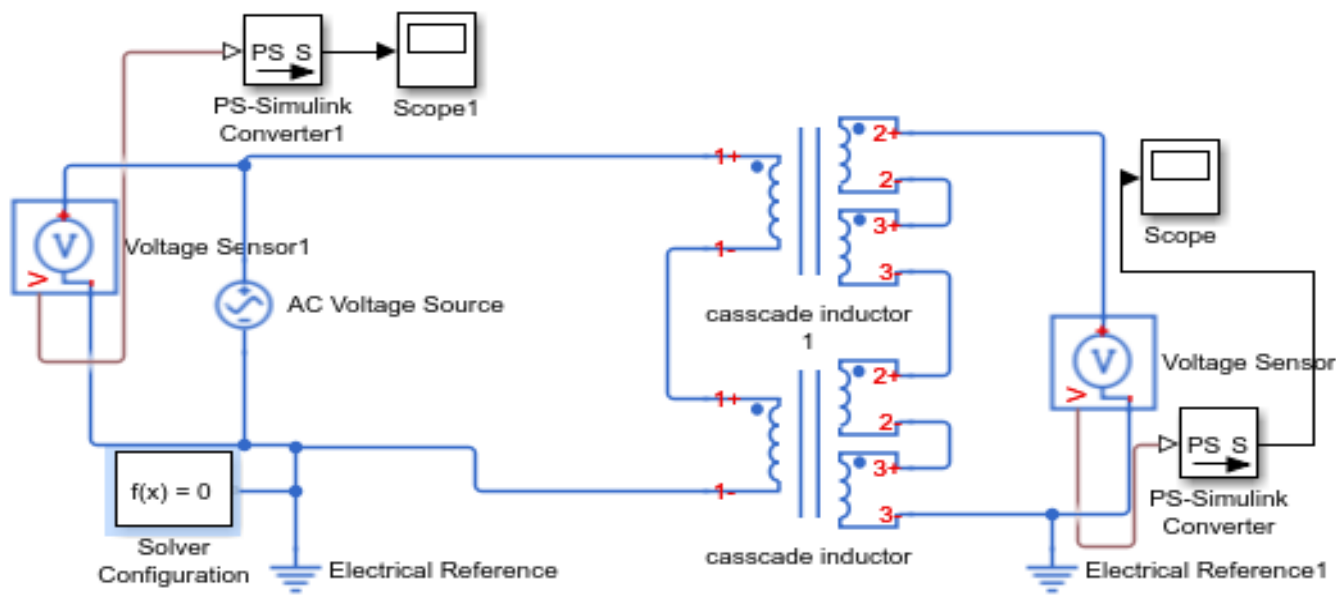

Figure 5. The figure shows the circuit of WPT with cascade coupling inductor

Table 2. Coefficient and output voltage

\begin{tabular}{|c|c|c|c|c|c|c|c|c|c|}
\hline Coefficient & 0.1 & 0.2 & 0.3 & 0.4 & 0.5 & 0.6 & 0.7 & 0.8 & 0.9 \\
\hline V out $(\mathrm{V})$ & 1 & 2 & 3 & 4 & 5 & 6 & 7 & 8 & 9 \\
\hline
\end{tabular}




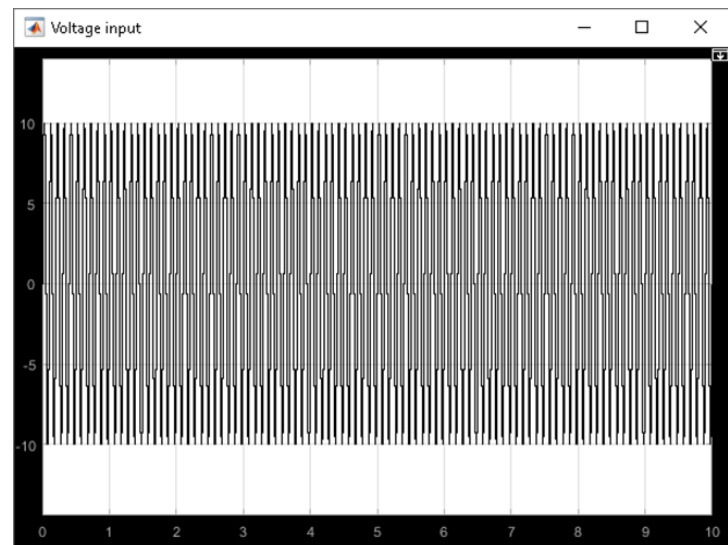

Figure 6. Voltage input

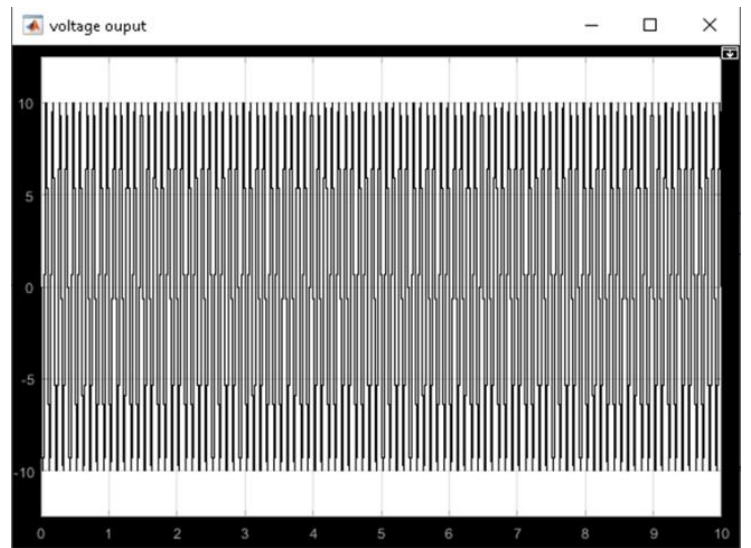

Figure 7. Voltage output

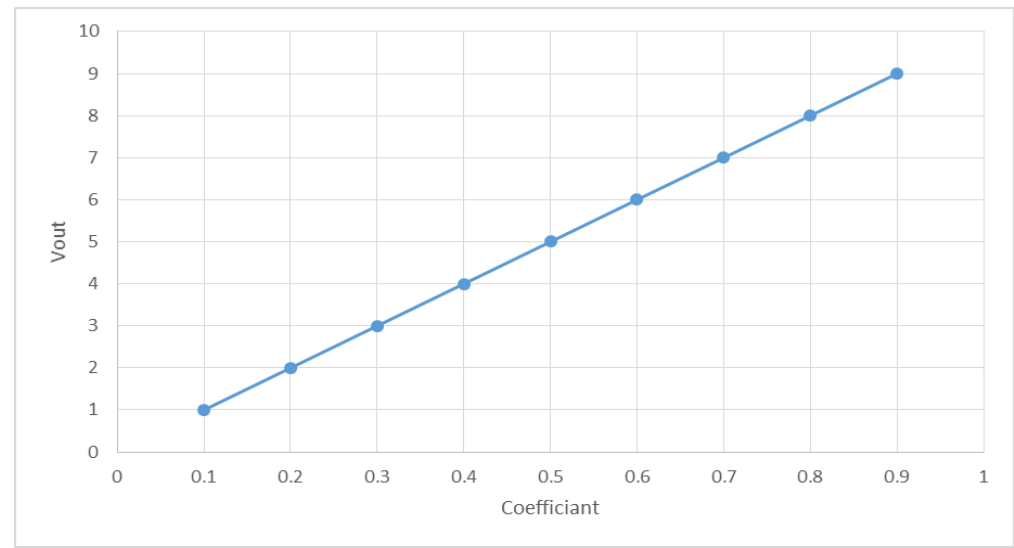

Figure 8. Coefficient and output voltage graph

\section{CONCLUSION}

Recently, wireless power transfer has got more attention from researchers and inventors because this technology has opportunity to change many things in the daily lifestyle as well as the industrial sector. In this research, wireless power transfer by using inductive coupling has been successfully simulated by using MATLAB Simulink. Parameters like self-inductance, mutual inductance and coupling coefficient are analytically determined for a pair of helical coils. The results of inductance calculation and their comparison with the experimental results showed that the inter-turn flux leakage could not be neglected. To improve the efficiency for this research, the transformer was implanted into the receiver circuit. To ensure the designed circuit does not get impacted by the heat that is produced by the coil, the cascade inductor was designed into the circuit as a coolant. Promising results have been obtained, the results indicated that, wireless power transfer can be considered as a solution for many situations where the power has to be transmitted wirelessly.

\section{ACKNOWLEDGEMENT}

The authors would like to express their gratitude to Universiti Kuala Lumpur for supporting and funding this research under grant No. str18005.

\section{REFERENCES}

[1] Mahmood, A. Ismail, Z. Zaman, H. Fakhar, Z. Najam, M. Hasan, et al., "A comparative study of wireless power transmission techniques," Journal of Basic and Applied Scientific Research, vol. 4, pp. 321-326, 2014.

[2] Alhamrouni, W. I. Hanis, M. Salem, F. M. Albatsh, and B. Ismail, "Application of DC-DC converter for EV battery charger using PWM technique and hybrid resonant," in 2016 IEEE International Conference on Power and Energy (PECon), pp. 133-138, 2016. 
[3] Ahmad, M. S. Alam, R. Chabaan, and A. Mohamed, "Comparative Analysis of Power Pad for Wireless Charging of Electric Vehicles," SAE Technical Paper 0148-7191, 2019.

[4] M. H. Alsharif, K. Yahya, and Z. W. Geem, "Strategic Market Growth and Policy Recommendations for Sustainable Solar Energy Deployment in South Korea," Journal of Electrical Engineering \& Technology, pp. 1-13, 2019.

[5] K. Yahya, M. Z. Bilgin, and T. Erfidan, "Practical implementation of maximum power tracking based short-current pulse method for thermoelectric generators systems," Journal of Power Electronics, vol. 18, pp. 1201-1210, 2018.

[6] A. Mohamed and O. Mohammed, "Bilayer Predictive Power Flow Controller for Bidirectional Operation of Wirelessly Connected Electric Vehicles," IEEE Transactions on Industry Applications, vol. 55, pp. 4258-4267, 2019.

[7] V.-B. Vu, V.-T. Phan, M. Dahidah, and V. Pickert, "Multiple output inductive charger for electric vehicles," IEEE Transactions on Power Electronics, vol. 34, pp. 7350-7368, 2018.

[8] V.-B. Vu, M. Dahidah, V. Pickert, and V.-T. Phan, "A High-Power Multiphase Wireless Dynamic Charging System with low Output Power Pulsation for Electric Vehicles," IEEE Journal of Emerging and Selected Topics in Power Electronics, 2019.

[9] E. L. Mathew and Y. P. Singh, "An Extensive study of "Wireless Power Transmission"-A Next Generation Power Transmission System.

[10] S. Gollakota, V. Talla, B. Kellogg, B. Ransford, S. Naderiparizi, and J. R. Smith, "Power transmission using wireless communication signals," ed: Google Patents, 2017.

[11] M. Salem, A. Jusoh, N. Rumzi, N. Idris, and I. Alhamrouni, "Steady state and generalized state space averaging analysis of the series resonant converter," 3rd IET International Conference on Clean Energy and Technology (CEAT) 2014, 2014.

[12] Q. Yuan, Q. Chen, L. Li, and K. Sawaya, "Numerical analysis on transmission efficiency of evanescent resonant coupling wireless power transfer system," IEEE transactions on antennas and propagation, vol. 58, pp. 1751-1758, 2010.

[13] M. Salem, V. K. Ramachandaramurthy, P. Sanjeevikumar, Z. Leonowicz, and V. Yaramasu, "Full Bridge LLC Resonant Three-Phase Interleaved Multi Converter For HV Applications," in 2019 IEEE International Conference on Environment and Electrical Engineering and 2019 IEEE Industrial and Commercial Power Systems Europe (EEEIC/I\&CPS Europe), pp. 1-6, 2019.

[14] M. Salem, V. K. Ramachandaramurthy, A. Jusoh, S. Padmanaban, M. Kamarol, J. Teh, et al., "Three-Phase Series Resonant DC-DC Boost Converter with Double LLC Resonant Tanks and Variable Frequency Control," IEEE Access, vol. 8, pp. 22386-22399, 2020.

[15] M. Salem, A. Jusoh, N. R. N. Idris, and I. Alhamrouni, "A review of an inductive power transfer system for EV battery charger," European Journal of Scientific Research, vol. 134, pp. 41-56, 2015.

[16] M. S. Issa, A. Onsy, M. S. Hamad, and A. El-Zonkoly, "Design of Wireless Power Charging System for Driverless Pod Application," in 2018 24th International Conference on Automation and Computing (ICAC), pp. 1-6, 2018.

[17] M. M. El Rayes, G. Nagib, and W. Abdelaal, "A review on wireless power transfer," International Journal of Engineering Trends and Technology (IJETT), vol. 40, pp. 272-280, 2016.

[18] S. K. Singh, T. Hasarmani, and R. Holmukhe, "Wireless transmission of electrical power overview of recent research \& development," International Journal of Computer and Electrical Engineering, vol. 4, p. 207, 2012.

[19] M. Salem, A. Jusoh, N. R. N. Idris, T. Sutikno, and Y. M. Y. Buswig, "Phase-shifted series resonant converter with zero voltage switching turn-on and variable frequency control," International Journal of Power Electronics and Drive Systems (IJPEDS), vol. 8, pp. 1184-1192, 2017.

[20] M. Salem, A. Jusoh, N. R. N. Idris, and I. Alhamrouni, "Comparison of LCL resonant converter with fixed frequency, and variable frequency controllers," in 2017 IEEE Conference on Energy Conversion (CENCON), pp. 84-89, 2017.

[21] Alhamrouni, M. B. Hamzah, M. Salem, A. Jusoh, A. B. Khairuddin, and T. Sutikno, "A bidirectional resonant converter based on wide input range and high efficiency for photovoltaic application," International Journal of Power Electronics and Drive System (IJPEDS), vol. 10, pp. 1469-1475, 2019.

[22] M. Salem, A. Jusoh, N. R. N. Idris, C. W. Tan, and I. Alhamrouni, "Phase-shifted series resonant DC-DC converter for wide load variations using variable frequency control," in 2017 IEEE Conference on Energy Conversion (CENCON), pp. 329-333, 2017.

[23] Alhamroun, M. Salem, A. Jusoh, N. R. N. Idris, B. Ismail, and F. M. Albatsh, "Comparison of two and four switches inverter feeding series resonant converter," in 2017 IEEE Conference on Energy Conversion (CENCON), pp. 334-338, 2017.

[24] M. Salem, A. Jusoh, N. R. N. Idris, H. S. Das, and I. Alhamrouni, "Resonant power converters with respect to passive storage (LC) elements and control techniques-An overview," Renewable and Sustainable Energy Reviews, vol. 91, pp. 504-520, 2018.

[25] V. Choudhary, S. P. Singh, V. Kumar, and D. Prashar, "Wireless power transmission: an innovative idea," International Journal of Educational Planning \& Administration, vol. 1, pp. 203-210, 2011.

[26] R. K. Jha, G. Buja, M. Bertoluzzo, S. Giacomuzzi, and K. N. Mude, "Performance comparison of the one-element resonant EV wireless battery chargers," IEEE Transactions on Industry Applications, vol. 54, pp. 2471-2482, 2018.

[27] M. Bertoluzzo, G. Buja, and M. Forato, "Transferred Power Leveling/Energy Maximization in Dynamic WPT Systems," in IECON 2018-44th Annual Conference of the IEEE Industrial Electronics Society, pp. 4856-4861, 2018. 